\title{
EL ERROR COMO OPORTUNIDAD: UN SIGLO DE REGISTRO Y EVOLUCIÓN DE LA MÚSICA POPULAR
}

\author{
Jon Martín Colorado \\ Universidad del País Vasco / Euskal Herriko Unibertsitatea. Dpto Dibujo
}

\section{Resumen}

El surgimiento y el desarrollo del registro de sonido ha incidido de manera evidente en la propia forma de la música popular; desde los campos rurales de principios del siglo $\mathrm{XX}$, hasta las ciudades industriales de los años 70 y las habitaciones de nuestras casas en la actualidad. Grabaciones de campo con una relación directa con el espacio y poco control sobre él; grabaciones de estudio donde se prescinde de micrófonos; grabaciones caseras donde se prescinde de estudio. Evolución en la cual se ha desarrollado asimismo una relación distinta respecto al concepto de la propia música y sus aspectos en cuanto a fidelidad, energía y error.

\footnotetext{
Palabras clave: MÚSICA; REGISTRO; FORMATO; FRAGMENTACIÓN; ERROR
}

\section{ERROR AS OPPORTUNITY: A CENTURY OF RECORDING AND EVOLUTION OF POPULAR MUSIC}

\section{Abstract}

The emergence and development of sound recording has had an obvious impact on the very form of popular music; from the rural fields of the early twentieth century, to the industrial cities of the 1970s and the bedrooms of our homes today. Field recordings with a direct relationship to space and little control over it; studio recordings where microphones are dispensed with; home recordings where the studio is dispensed with. Evolution in which a different relationship has also developed in respect to the concept of music itself and its aspects in terms of fidelity, energy and error.

Keywords: MUSIC; RECORDING; FORMAT; FRAGMENTATION; ERROR

\footnotetext{
Martín Colorado, Jon. 2021. "El error como oportunidad: Un siglo de registro y evolución de la música popular". AusArt 9 (1): 173-183. D0I: 10.1387 /ausart.22671
}

\section{AUSART}




\section{CAMPOS DE TRABAJO Y PRIMERAS GRABACIONES DE CAMPO}

No es lo mismo escuchar un sonido grabado y escuchar ese mismo sonido directamente desde su fuente. Lo mismo da usar micrófono, enchufar el instrumento directamente a la grabadora o generarlo mediante sintetizador, ordenador, etc. Registrar un sonido es manipularlo. Ciertamente, ni siquiera el propio sonido tiene forma preconcebida alguna; se amolda a su entorno constantemente, rebotando, vibrando, reverberando con todo lo que se encuentra en él. No hay más que dar una palmada en una habitación amueblada o en una vacía, con las paredes de madera o cemento, al aire libre, etc. El sonido es así reflejo del espacio, y por lo tanto, parte también de su diseño, reconocible en la arquitectura y, en principio, más cuidado en sitios como auditorios y salas de grabación o conciertos.

Desde que Thomas Edison realizara el primer registro de sonido en 1877 (Gioia 2010, 71), la evolución de las técnicas de grabación ha ido modificando la relación que tiene con el espacio. Se trata de un camino que va desde lo acústico a lo eléctrico y de lo eléctrico a lo electrónico y digital; desde el blues de los esclavos en los campos de algodón del delta del río Mississippi, a la música industrial de las ciudades y entornos de las fábricas de Inglaterra en los años 70 y las habitaciones de nuestras casas en la actualidad.

Durante la primera década del siglo XX, W. Odum realizó las primeras grabaciones de campo estudiando las músicas populares folk de la época. A éstas les siguieron las realizadas por Henry Spier y, sobre todo, las más relevantes realizadas por John Lomax y su hijo Alan para la Biblioteca del Congreso de los Estados Unidos (Cowey 1994, 265). De la misma manera que el resto de músicas antes de su comercialización en formato físico, el blues del delta del Mississippi estaba fuertemente unido a la vida diaria de la comunidad; cantos religiosos, de trabajo... que se transmitían de generación en generación y que, evidentemente, no estaban pensadas para grabarse. "Cantaban canciones de forma improvisada y con acompañamiento de guitarra sobre la vida de cada día, el amor, la mala suerte y los buenos tiempos" (Evans 1994, 34).

Esas primeras grabaciones se realizaban colocando un primitivo cono enfrente del sonido que se quería captar. El micrófono no trajo mayores cambios en ese sentido; voz, palmas, golpes en el suelo o instrumento acústico del que se tratase seguían capturándose al unísono. La elección del espacio y la colocación del micrófono y los músicos —en relación al volumen al que sus instrumentos 
iban a ser captados - eran fundamentales en el resultado. Robert Johnson, que se ha convertido en la leyenda del género por excelencia, es el protagonista de varias anécdotas y leyendas. Cuentan que al grabar se colocaba tocando de cara a la pared, en una esquina de la habitación, como muestra la ilustración de la portada de uno de sus discos (King of the Delta Blues Singers Vol. II, New York: Columbia Records, 1970; la anécdota aparece también ilustrada en el cómic Love in vain de Mezzo \& Dupont). Ya fuera por timidez o lo hiciera conscientemente, la influencia en el sonido de la voz y la guitarra resulta innegable.

Además de por el registro, la música está también condicionada por el soporte físico de la edición: "la historia del blues es inseparable de la del disco fonográfico, que ha desempeñado un papel crucial en la difusión de la música popular en este siglo" (Spottswood 1994, 88). El límite físico del disco de pizarra, vinilo, CD... afecta directamente a la duración del contenido, haciendo que las canciones se amolden a menudo al mismo. El caso de Robert Johnson vuelve a ser muy ilustrativo en este caso; las 29 canciones que forman su legado completo, registradas durante dos sesiones en 1936 y 1937, coinciden exactamente con el límite de la duración máxima - entre dos y tres minutos- que permitía el disco de pizarra de diez pulgadas a 78 revoluciones por minuto en el que fueron editadas.

"En los discos de 78 RPM sólo cabían unos minutos de música y se avisaba a los intérpretes con una luz roja cuando el tiempo estaba acabando. A veces, los novatos se quedaban inmóviles cuando veían la luz, y dejaban de tocar de inmediato. En otros casos, la interpretación concluía a tiempo, pero una perceptible aceleración en la última vuelta quedaba como testimonio de los nervios que pasaban los músicos. Incluso en las circunstancias ideales, el ambiente esterilizado de los estudios, sin bailarines ni público que inspirara a los artistas, sólo con el personal contratado que miraba una y otra vez el reloj y se ocupaba sin compasión de los detalles, tendía a reprimir la vitalidad de la música."

(Gioia 2010, 80).

Cuando el vinilo remplazó a la pizarra, el tamaño y la velocidad se redujeron, pero se mantuvo la misma duración: desde entonces, el disco de 7 pulgadas a 45 RPM estableció el concepto de single, todavía vigente en la actualidad.

Además de los autoimpuestos, los límites son, obviamente, naturales. Los seres humanos nos hemos tenido que amoldar a ellos ininterrumpidamente, 
basando nuestro saber en la observación, experiencia y experimentación en la naturaleza. Ambos son mutables, naturaleza y conocimiento. No pueden ser definitivos. Los sistemas orgánicos se caracterizan por su movilidad, sus cambios y sus límites. Los factores son innumerables y se afectan-adaptan constantemente entre sí. El clima es un buen ejemplo de esto; cambiante e imposible de predecir con gran antelación, se comporta como lo hiciera lo que habitualmente entendemos como organismo vivo autónomo. El concepto de organismo es por lo tanto flexible; abarca fenómenos de diferente complejidad como una célula, un animal o un hormiguero. Depende de la escala desde la que se observa.

\section{INDUSTRIALIZACIÓN Y FRAGMENTACIÓN}

"La naturaleza está en cualquier lugar en perpetuo movimiento: las condiciones no son nunca exactamente las mismas de un año a otro. La investigación moderna divide a la naturaleza en pequeños pedazos y realiza experimentos que no se ajustan ni a las leyes naturales ni a la experiencia práctica. Los resultados se ordenan según la conveniencia del investigador, no de acuerdo con las necesidades del agricultor. Creer que estas conclusiones pueden utilizarse con éxito invariable en la parcela del agricultor es un gran error. (...) La geografía y topografía del lugar, el estado del suelo, su estructura, textura y drenaje, exposición al sol, relaciones con los insectos, la variedad de semilla utilizada, el método de cultivo - verdaderamente una infinita variedad de factores-deben ser considerados. Un método de ensayo científico que tenga en cuenta todos los factores relevantes es imposible."

(Fukuoka [1975] 2011, 81)

Basados a menudo en los procesos orgánicos, los artefactos, a falta de órganos, están formados por piezas y tienen funciones concretas que deben cumplir de manera precisa. Una piedra de sílex corta lo que no pueden los dientes, una lanza alcanza y hiere lo que no pueden nuestras piernas y manos. Son, por lo tanto, extensiones del cuerpo; "La técnica comenzó cuando el hombre usó por primera vez sus dedos como pinzas o una piedra como proyectil: como el arte mismo, se enraíza en el uso que el hombre hace de su propio cuerpo" (Mumford [1952] 1968, 21). Son antítesis del clima, por seguir con un ejemplo 
anterior; forman parte del mundo físico y por lo tanto son afectados por el entorno; antes o después se estropean, se oxidan... pero su mecanismo es normalmente un orden cerrado, autónomo, inalterable, impermeable.

En cualquier caso, a partir del siglo XIX, y con el apoyo de pensadores avanzados, la máquina se apropió de todos los aspectos de la vida, convirtiéndose en el fundamento principal que daba sentido a la existencia humana. " $L a$ potencia, la velocidad, la estandarización, la movilidad, la producción en masa, la cuantificación, la regimentación, la uniformidad, la precisión, la regularidad astronómica y el control -sobre todo, el control- pasaron a ser contraseñas de la sociedad occidental moderna" (Mumford [1967] 2010, 480).

Poder, Beneficio y Prestigio son, según Mumford, valores basados en los tres cánones principales del capitalismo: "el cálculo de la cantidad, la observación y regimentación del tiempo ('Time is money') y la concentración en gratificaciones pecuniarias abstractas" (ibíd., 459). Las tareas de los obreros de las fábricas empezaron a medirse y controlarse por medio del cronómetro, intentando optimizarlas hasta en milésimas de segundo (Rifkin [1995] 2008, 105). El esclavo de las plantaciones de algodón se convierte así en el trabajador libre, pero sarcástico, de las ciudades industriales, y siguiendo a éste, sus cantos de trabajo se transforman igualmente.

Los precursores de la música industrial, o por lo menos aquellos que la bautizaron, fueron Throbbing Gristle, a mediados de los años 70 en Yorkshire, Reino unido. Éstos procedían de un movimiento artístico llamado COUM Transmissions (P-Orridge 2009, 29.), en el que recuperaban el espíritu provocador de las vanguardias, con sucesivas acciones y la publicación manifiestos; el surrealismo de Dalí, el absurdo de Alfred Jarry, y sobre todo, con la actitud y técnicas del dadaísmo de Tristan Tzara. Musicalmente se entrevén influencias de Frank Zappa, Captain Beefheart, Edgar Varèse o lannis Xenakis.

Este tipo de música acostumbra a ser mayormente electrónica, de modo que los micrófonos son a menudo prescindibles y el sonido es creado directamente con sintetizadores, simuladores. En los créditos del disco Dreams less sweet (London: Some Bizzare ¡CBS, 1983.), Psychic TV ¡-grupo formado por miembros de Throbbing Gristle- se jactaban de no usar ningún tipo de micrófono y haber registrado el sonido mediante los métodos de grabación holofónica de Zuccarelli Holophonics. Un ejemplo bien diferente sería el de los alemanes Einstürzende Neubauten, que en la contraportada de Kollaps (Hamburg: Zickzack records, 1981) -y parodiando la del Ummagumma de Pink Floyd 
(London: Harvest records, 1969) - mostraban el equipo que usado para crear su música: un par de amplificadores, una guitarra, pedales de efectos, teclados, un taladro, un martillo neumático, un megáfono, una guitarra de juguete, una hacha, cachos de cañerías, una llave de perro, un martillo, una sierra, una televisión, chapas y tapas de metal, un rastrillo, etc. En este sentido, el espacio ya no forma necesariamente parte del sonido o pierde, por lo menos, gran parte de su influencia sobre él. En una clara referencia al funcionamiento de las máquinas, sus ritmos y estructuras son bastante repetitivas - normalmente bases programadas con cajas de ritmos-. Algo similar ocurre con las letras de sus canciones - cuando las hay-; o bien se repiten en tono robótico, o bien transmiten mensajes fragmentados sin sentido aparente, en una clara aplicación de la técnica de recortes que Tristan Tzara describe en uno de los manifiestos del dadaísmo:

"Para hacer un poema dadaísta

Coja un periódico.

Coja unas tijeras.

Escoja en el periódico un artículo de la longitud que cuenta darle a su poema.

Recorte el artículo.

Recorte en seguida con cuidado cada una de las palabras que forman el artículo y métalas en una bolsa.

Agítela suavemente.

Ahora saque cada recorte uno tras otro.

Copie concienzudamente

en el orden en que hayan salido de la bolsa.

El poema se parecerá a usted.

$Y$ es usted un escritor infinitamente original y de una sensibilidad hechizante, aunque incomprendida del vulgo"

(Tzara [1924] 1979, 50)

El dadaísmo fue una gran influencia para el movimiento surrealista. Vemos así como la técnica de recortes del primero recuerda tanto al collage, como al azar y el automatismo del segundo. Throbbing Gristle empezaron a utilizarlo en realidad por influencia del escritor William Burroughs, que lo había tomado a su vez del pintor Bryon Gysin. Para ellos se trataba de una manera de hacer frente al "virus del lenguaje", un concepto lineal que nos hace aceptar la realidad como un camino único entre dos puntos, una forma de control que manipula el comportamiento humano, negando la intuición y, particularmente, los instintos y los sueños. Mediante el uso de este método su intención era, precisamente, "cortocircuitar" ese control, consiguiendo un acercamiento más fiel 
a la realidad tal y como es (P-Orridge 2009, 131). "Todo en la vida es recorte"; partiendo de la información que recibe a través de los sentidos, nuestro cerebro codifica y construye constantemente "una imagen manejable del mundo"; reduce su complejidad e impone una linealidad para facilitar la comunicación básica y la supervivencia. "Lo que vemos, lo que oímos, lo que olemos, lo que tocamos, lo que sentimos, lo que decimos, son aproximaciones aplanadas y apagadas de una mucho más intensa, vibrante y caleidoscópica realidad ultra-dimensional" (ibíd., 147).

Huxley considera el lenguaje y los sistemas de símbolos imprescindibles en nuestro desarrollo como seres humanos. Sin embargo, del mismo modo que al deformar los hechos para volverlos familiares, las palabras y los conceptos son beneficiosos, en ocasiones pueden resultar también una traba a la hora de mirar y conocer el mundo de manera directa. Además de ser conscientes del mismo, el razonamiento sistemático tendría que ser, según él, compatible con una percepción cuanto menos sistematizada mejor ( [1954] 1992, 71).

La música industrial muestra el mismo interés por la fragmentación en la manera de generar sonidos. Utiliza, por ejemplo, herramientas como la síntesis granular desarrollada por lannis Xenakis. Partiendo de la concepción del sonido como una serie de partículas o granos elementales - concretamente alrededor de 340.000 audibles-, Xenakis proponía la posibilidad de construir cualquier sonido posible dentro de los límites humanos: manipulaciones que podrían producir tanto sonidos conocidos como los de los instrumentos clásicos, cuerpos elásticos o aquellos referidos a la música concreta, así como perturbaciones sónicas con evoluciones, no paralelas e inimaginables hasta el momento. "La base de las estructuras y transformaciones los timbres no tendrá nada que ver con lo que se ha conocido hasta ahora" ( [1963] 1992, 47).

\section{ERROR Y OPORTUNIDAD}

"La música del mañana, al proceder por una estructuración inédita, particular, del espacio, y del tiempo, podría convertirse en una herramienta de transformación del hombre, al influir sobre su estructura mental" 
En las composiciones de sus trabajos musicales y de arquitectura, Xenakis ahondó en la misma idea mediante el uso del concepto de modulor - módulo-. Así podemos apreciarlo en la fachada acristalada del convento de La Tourette (L'Arbresle, Francia), una de sus obras más reseñables mientras trabajó en el estudio de Le Corbusier. Su intención era crear ritmos musicales en la misma, para lo cual ideó incluso un sistema basado en la sección aurea que denominó ondulatories - por sugerencia de Le Corbusier, ya que su primera idea era musical glass surfaces-(Xirou 2009, 18m). Del mismo modo realizó algunas composiciones para orquesta como Metastaseis o Pithoprakta, formada por 46 instrumentos de cuerda con partituras individuales, donde cada instrumento se comporta como una molécula de gas en el espacio (Xenakis [1963] 1992, 15).

Es también digna de mención la versión de Vexations realizada por John Cage, en la que 10 pianistas se turnaban entre sí para repetir 840 veces, sin parar durante 18 horas y 40 minutos, un módulo de 1,20 minutos de la obra original de Erik Satie. Más que potenciar el significado de similitud entre sí, las consecutivas repeticiones evocan, según Ariza, "un concepto de repetición expandido bajo el significado de variación" (Ariza 2003, 49).

Por su parte, el registro de la música, con la máquina como modelo, se fue modificando también hacia la fragmentación. La grabadora permitió el uso de varios micrófonos simultáneamente en una misma cinta, y pronto la posibilidad de grabar pistas en tiempos distintos. A pesar de ello, todavía resulta posible escuchar a los músicos tocando juntos en muchas grabaciones de los años 70; tal vez porque entendían el sonido como el resultado directo de la energía y la retroalimentación de una ejecución simultánea. Las sesiones de grabación del Funhouse de The Stooges son un ejemplo inmejorable en este punto; cerca de 8 horas tocando 142 variaciones distintas de las mismas 7 canciones (The Stooges. 1970: The Complete Funhouse Sessions. Rhino Handmade, 1999).

La tendencia general y que se mantiene hasta nuestros días es, sin embargo, la de grabar a cada músico tocando de manera aislada. Esto permite corregir más fácilmente cada instrumento sin afectar al conjunto. Eso que se busca es, precisamente, lo que se pierde: la retroalimentación de varios micrófonos y sonidos simultáneos, en beneficio del sonido limpio. Para mantener la coherencia natural del directo surge, entonces, la necesidad de usar el metrónomo. Su ritmo frío y mecánico se convierte en guía para todos los músicos, y la propia música, en una suma de fragmentos. 
En las últimas décadas, la grabación digital permite, por ejemplo, capturar el golpe de una batería y procesarlo como le apetezca a uno, lo que se conoce como disparar un sonido o usar triggers. Esta tecnología ofrece, incluso, la posibilidad de cambiar la ubicación de esos golpes en la línea de tiempo de la canción. Esto se usa, normalmente, con la intención de corregir una ejecución que no se ajusta a la cuadratura perfecta del metrónomo, lo que se conoce como cuantizar la batería, ajustar el golpe al nanosegundo que le corresponde. Hechos como estos, en los que el efecto en el resultado ni siquiera es claramente perceptible, dejan al descubierto una especie de obsesión por adaptarse a un modelo que se toma como teóricamente perfecto.

Al mismo tiempo, sin embargo, han surgido quiénes han sabido aprovechar ese error como un recurso plástico, sonoro, conceptual y expresivo. Siguiendo los pasos de la música electrónica e industrial nos encontramos a artistas como Alva Noto, Autechre, Demdike Stare o JASSS, con su uso del glitch, las estructuras rotas, saturación de sonidos o errores de formato, ya sea mediante loops, discos de vinilo rayados o incluso simulaciones de error de lectura de archivos digitales.

Si bien la música electrónica es sin duda la vanguardia de ese tipo de aprovechamiento artístico del error, también se da en otros estilos musicales urbanos. Ya a finales de los años 80 el death metal tuvo que enfrentarse a esa concepción perfeccionista y limpia de los ingenieros de sonido, con unos primeros resultados que todos los participantes consideraron inmediatamente aberrantes; valga de ejemplo el primer álbum de unos de los creadores del grindcore junto a Napalm Death: Carcass y su Reek of Putrefaction (Earache records, 1988). Afortunadamente, otros como Burzum y Dark Throne encontraron mayor comprensión y cooperación en la Noruega de 1992, con enfoques y resultados que esta vez sí, aportaron de manera definitiva a un registro más fiel del ruido y la suciedad. A diferentes marchas músicos, productores e ingenieros de sonido han seguido evolucionando (Blasphemy, Ulver, Incantation, Mortician, Zanussi...), llegando a extremos que siguen sucediéndose hasta el día de hoy, como el caso de los madrileños Teitanblood y la nota promocional que acompañaba a su álbum Death del año 2014: "The second Teitanblood album corrects the misconception about death metal being music. Mortui vivos docent".

El caso de la música trap es seguramente el ejemplo más ilustrativo de toda esta evolución: grabaciones caseras en habitaciones de adolescentes, con recursos materiales limitados, pero suficientes para permitir crear cómoda- 
mente (y desde luego infinitamente más versátiles que los 4 pistas analógicos de la época del hardcore y punk de los años 70 y 80); y sobre todo, la apropiación de herramientas como el autotune, pensadas en un principio para corregir errores y que son ahora seña de identidad sin de este estilo musical. Distintas concepciones del error que sirven así como motor para que la música siga evolucionando y reinventándose.

\section{Referencias bibliográficas}

Ariza Pomareta, Javier. 2003. Las imágenes del sonido: Una lectura plurisensorial en el arte del siglo XX. Cuenca: Universidad de Castilla-La Mancha

Cowey, John H. 1994. "No me dejes aquí: El blues no comercial; las grabaciones de campo, 1924-1960". En Solamente blues: La música y sus músicos, Mary Katherine Aldin et al.; Lawrence Cohn, comp.; traducción, Yolanda Fontal. Barcelona: Odín

Evans, David. 1994. "Recorriendo el país: El blues de Texas y el profundo Sur". En Solamente blues: La música y sus músicos, Mary Katherine Aldin et al.; Lawrence Cohn, comp.; traducción, Yolanda Fontal. Barcelona: Odín

Fukuoka, Masanobu. (1975) 2011. La revolución de una brizna de paja: Una introducción a la agricultura natural. Teruel: EcoHabitar

Gioia, Ted. 2010. Blues: La música del Delta del Mississippi. Ilustraciones de Neil Harpe, traducción de Mariano Peyrou. Madrid: Turner

Huxley, Aldous. (1954) 1992. Las puertas de la percepción; Cielo e infierno. Traducción de Miguel de Hernani. Barcelona: Edhasa

López, Julio. 1984. La música de la modernidad: De Beethoven a Xenakis. Barcelona: Anthropos

Mumford, Lewis. (1952) 1968. Arte y técnica. Traducción de Luis Fabricant. Buenos Aires: Nueva Visión

Mumford, Lewis. (1967) 2010. El mito de la máquina: Técnica y evolución humana. Traducción, Javier Rodríguez Hidalgo. Logroño: Pepitas de Calabaza

P-Orridge, Genesis Breyer. 2009. Thee psychick bible. New York: Feral House

Rifkin, Jeremy. (1995) 2008. El fin del trabajo: El declive de la fuerza de trabajo global y el nacimiento de la era posmercado. Prólogo de Robert L. Heilbroner; traducción de Guillermo Sánchez. Barcelona: Paidós

Spottswood, Richard. 1994. "Chicas del campo, blues clásico y voces de variedades. Las mujeres y el blues". En Solamente blues: La música y sus músicos, Mary Katherine Aldin et al.; Lawrence Cohn, comp.; traducción, Yolanda Fontal. Barcelona: Odín

Tzara, Tristan. (1924) 1979. Siete manifiestos Dada. Con algunos dibujos de Picabia; traducción de Huberto Haltter Barcelona: Tusquets

Xenakis, lannis. (1963) 1992. Formalized music: Thought and mathematics in composition. Stuyvesant NY: Pendragon 
Xirou, Efi. 2009. Charisma X: Iannis Xenakis. New York: Mode Records, DVD, 61 min.

(Artículo recibido: 28-03-21; aceptado: 04-06-21) 